\title{
Revealing the importance of interfaces for pure spin current transport
}

\author{
A. Pfeiffer, ${ }^{1,2}$ R. M. Reeve $\odot,{ }^{1,2}$ K. Elphick, ${ }^{3}$ A. Hirohata $\odot,{ }^{3}$ and M. Kläui $\odot^{1,2, *}$ \\ ${ }^{1}$ Institut für Physik, Johannes Gutenberg Universität Mainz, 55099 Mainz, Germany \\ ${ }^{2}$ Graduate School of Excellence Materials Science in Mainz,(MAINZ), Staudinger Weg 9, 55128 Mainz, Germany \\ ${ }^{3}$ Department of Electronic Engineering, University of York, Heslington, YO10 5DD York, United Kingdom
}

(Received 1 December 2019; revised 14 April 2021; accepted 15 April 2021; published 10 May 2021)

\begin{abstract}
Spin transport phenomena underpin an extensive range of spintronic effects. In particular spin transport across interfaces occurs in most device concepts, but is so far poorly understood. As interface properties strongly impact spin transport, one needs to characterize and correlate them to the fabrication method. Here we investigate pure spin current transport across interfaces and connect this with imaging of the interfaces. We study the detection of pure spin currents via the inverse spin Hall effect in Pt and the related spin current absorption by $\mathrm{Pt}$ in $\mathrm{Py}-\mathrm{Cu}-\mathrm{Pt}$ lateral spin valves. Depending on the fabrication process, we either find a large (inverse) spin Hall effect signal and low spin absorption by Pt or vice versa and a similar effect is seen also for spin current absorption in $\mathrm{CoFe}-\mathrm{Cu}$-based devices. We explain these counterintuitive results by the fabrication-induced varying quality of the interfaces, which is directly revealed via a special scanning electron microscopy technique for interface imaging and correlated to the spin transport.
\end{abstract}

DOI: 10.1103/PhysRevResearch.3.023110

\section{INTRODUCTION}

Spintronics aims to harness the electronic spin degree of freedom for a wide range of potential device functionalities that are underpinned by spin transport through interfaces, such as magnetoresistance effects including giant magnetoresistance [1,2] and spin-torque manipulation of magnetic states [3-5]. While the structure and composition of the interfaces can be expected to strongly affect the spin transport [6-8], a direct characterization of the systems is challenging since the relevant interfaces are often buried under other layers. Hence they are not accessible with conventional surface-sensitive imaging approaches and transmission electron microscopy does not allow for quantification of the quality of large areas. It is well known that interface quality and in turn spin transport can be affected by the choice of materials and the growth modes [7,9,10]; however the role of other aspects of the fabrication procedure is poorly understood. In particular the choice of the patterning process may drastically modify the interfaces of the device. It is well known that spin scattering at interfaces can occur by the presence of interfacial contamination and changes to the interface morphology and such changes to the interfaces can also arise from the patterning. The resulting spin transport could be affected by the resulting differences in the material grain structure and interface structure [11,12], by the presence of contamination and oxidation [13-15], and

\footnotetext{
*klaeui@uni-mainz.de

Published by the American Physical Society under the terms of the Creative Commons Attribution 4.0 International license. Further distribution of this work must maintain attribution to the author(s) and the published article's title, journal citation, and DOI.
}

by the existence of voids and surfaces [16]. Yet without direct characterization of the interfaces the correlation between the fabrication method, interface quality, and measured spin transport remains largely unknown. Recently, however, a technique has been pioneered which provides direct nondestructive access to buried interface characterization via a scanning electron microscope (SEM) [17-19]. This technique opens up possibilities for such a direct connection between the spin transport and interfaces in relation to the fabrication recipes.

One particular prominent example where spin transport across interfaces plays a crucial role is the spin Hall effect (SHE), which provides the fascinating possibility to generate spin currents in nonmagnetic heavy metals (HMs) with large spin-orbit coupling [20,21]. If an electric charge current is flowing in such a HM, a spin current is generated perpendicular to this charge current, with a polarization given by the vector product of the charge and spin current propagation direction. The reciprocal inverse spin Hall effect (iSHE) describes the appearance of an electric charge current on spin current absorption in such SHE materials. In order to maximize spin transport across interfaces, in addition to the spin Hall angle $\theta_{\mathrm{SH}}$ describing spin to charge conversion [21], other contributions including spin transparency [7] and spin memory loss $[6,8,22]$ must be understood and optimized. In many studies such sources of spin relaxation are not considered, yet they can significantly affect the measured signals $[7,22]$. Spin transparency describes the relative transmission of different spin channels across the interface based on the spin mixing conductance [23]. Spin memory loss describes the partial depolarization of the spin current caused by spin-flip events as the spin current traverses the relevant interfaces.

Various techniques have been applied to determine $\theta_{\mathrm{SH}}$ experimentally including spin pumping [24-26], spin Hall 
magnetoresistance [27], and spin-orbit torque measurements [22]. However, even in the case of Pt, the most widely studied spin Hall material, the determined spin Hall angles differ considerably between 0.01 and 0.20 [7,28,29], depending on the particular study and the technique employed. These different techniques are based on different assumptions in particular concerning the nature of the relevant interfaces, and hence their validity and applicability need to be checked. One particularly widely applied method to study spin transport through interfaces is the spin absorption method in a lateral spin valve device [30-33]. In this geometry, two ferromagnetic (FM) electrodes are spatially separated but connected via a nonmagnetic (NM) bridge, which acts as a spin current conduit. The FM electrodes can be employed to inject and detect pure spin currents and if there is a further intermediate HM electrode present, additional spin current absorption by the HM can occur. By a comparison of the measured signals with and without a SHE intermediate electrode, as well as the generated iSHE voltage across the HM, models have been proposed to describe the spin transport and to determine the spin transport parameters of the materials, in particular the spin Hall angle $\theta_{\mathrm{SH}}$ and the spin diffusion length $\lambda_{\mathrm{HM}}$ of the HM [30]. However, in these and similar devices, the contribution of spin transparency and spin memory loss at the HM/NM and FM/NM interfaces and their connection to the interface structure is currently unclear. Of particular relevance is the role of the fabrication method in modifying spin transport via the resulting possible changes to the interfaces, including not only the material growth but moreover the often disregarded role of the patterning process. This, however requires a direct comparison between the quality of the interfaces and the spin transport, which is so far missing.

In this work we investigate the spin transport across $\mathrm{Cu} / \mathrm{Pt}$ and $\mathrm{Cu} / \mathrm{Py}$ interfaces using the spin absorption method in a multiterminal Pt-Py-Cu lateral spin valve. We fabricate two types of devices, for which different processes have been used to pattern the $\mathrm{Cu}$ bridge, leading to different interface properties and in particular different defect densities. For these two cases we compare the conventional nonlocal signal, the spin absorption, and the (inverse) spin Hall effect signal for varying temperatures. Depending on the sample fabrication method, we either find a very large (i)SHE signal and nearly no spin absorption or a low (i)SHE signal but strong spin absorption. The results are discussed in terms of the properties of the interfaces of the devices. To reveal the origin, the interfaces are imaged via a special scanning electron microscopy technique for buried interface characterization. Our findings demonstrate that the spin absorption method for determining spin transport parameters is not robust without further device characterization and highlight the sensitivity of spin transport to the interface properties that in turn are strongly governed by the fabrication method. Complementary measurements for $\mathrm{CoFe} / \mathrm{Cu}$-based devices are performed which support the results of the Pt-Py-Cu system.

\section{SAMPLE FABRICATION}

Lateral spin valve samples with a kinked geometry as shown in Fig. 1(a) are fabricated on a sapphire substrate by electron beam lithography (EBL) and liftoff techniques.
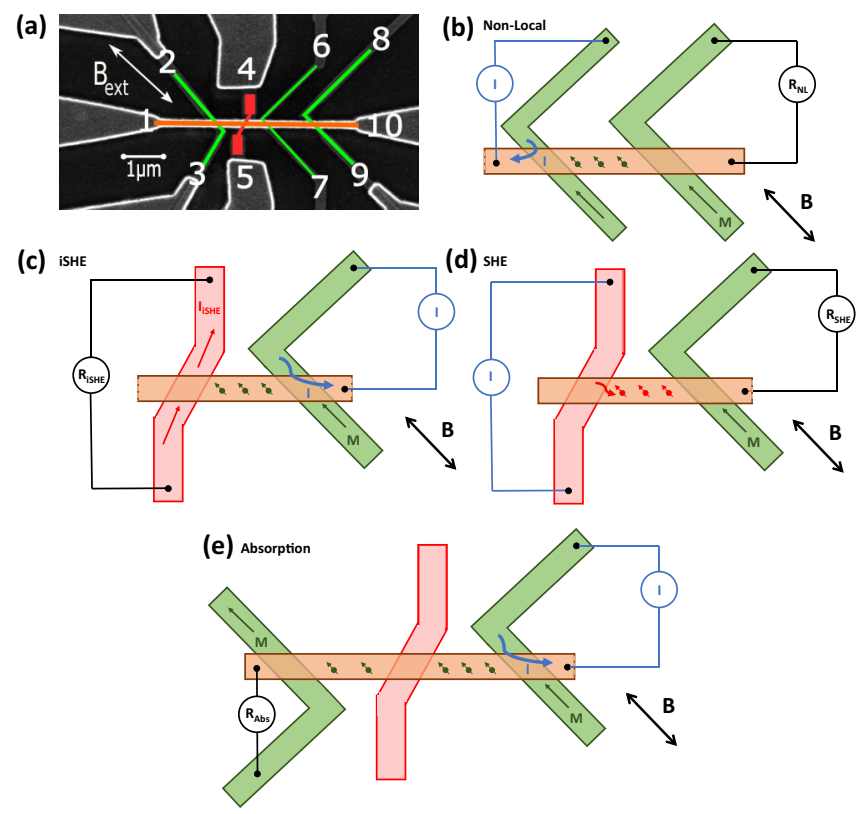

FIG. 1. (a) Annotated scanning electron microscope image of the sample with the wires color coded. For both recipes, the sample consists of one Pt stripe (drawn in red), three Py wires (drawn in green), and one $\mathrm{Cu}$ bridge (drawn in orange). (b)-(e) Schematic images of the main measurement modes. The wires have the same color code as in (a). The magnetization of the wires in the relevant regions is indicated by the large green arrows and the applied current by the blue arrows and blue current source. The generated (i)SHE current/spin current is indicated by the red arrows. The spin current orientation is indicated by the small circles with arrows. The signal contacts are depicted in black.

In the first step, a $100 \mathrm{~nm}$ wide and $1 \mu \mathrm{m}$ long stripe is patterned, together with alignment markers, and $16 \mathrm{~nm}$ of $\mathrm{Pt}$ is deposited using magnetron sputtering (red stripe). In the second step, three wires, one $140 \mathrm{~nm}$ and two $180 \mathrm{~nm}$ in width, are patterned perpendicularly to the Pt stripe and $25 \mathrm{~nm}$ of Py is deposited by ultrahigh vacuum (UHV) thermal evaporation (green wires). After the deposition and the liftoff processing of the Py wires, the substrate has been cut into two $5 \times 10 \mathrm{~mm}^{2}$ pieces. For the patterning of the nonmagnetic bridge, two different recipes have been used based on two different resist layers in order to investigate whether this change in the patterning influences the properties of the devices. These particular resists are chosen due to their wide employment in the literature. The first recipe is based on a standard poly(methyl methacrylate) (PMMA) resist whereas the second recipe is based on a double-layer methyl methacrylate (MMA)/PMMA resist. Such double-layer resists are also commonly employed since they yield a large undercut on development, which can assist in the liftoff process, in particular in the case of thick layers such as the copper conduit employed here. The details of the recipes are as follows:

Recipe 1. $300 \mathrm{~nm}$ of PMMA 950K A4 has been spun onto the chip and the EBL has been performed using $20 \mathrm{kV}$ acceleration voltage of the primary beam.

Recipe 2. First $150 \mathrm{~nm}$ of MMA EL6 has been spun and as a second resist, $300 \mathrm{~nm}$ of PMMA 950 A4 has been 
used. The EBL has been performed using $10 \mathrm{kV}$ acceleration voltage of the primary beam.

For both recipes, the baking time of the resist(s) is 90 seconds at $180^{\circ} \mathrm{C}$ on a hot plate. The development has been performed for both recipes using one part of methyl isobutyl ketone (MIBK) diluted in three parts of isopropyl alcohol (IPA) for 45 seconds. The used exposure doses for the two recipes have been independently optimized in order to yield low ohmic electric interface resistances in the $m \Omega$ range. In the last step, in situ argon milling is used to clean the interfaces of the Py wires and the Pt stripe for both recipes at the same time with the substrates mounted on the sample holder next to each other and with the same orientation with respect to the argon gun. Finally, a $170 \mathrm{~nm}$ wide (for recipe 1) and a $190 \mathrm{~nm}$ wide (for recipe 2) and $85 \mathrm{~nm}$ thick Cu bridge has been deposited via UHV thermal evaporation (orange wire in Fig. 1), together with electric contacts.

\section{COMPARISON OF THE NONLOCAL AND THE SPIN ABSORPTION SIGNAL FOR BOTH RECIPES}

Schematics of the different measurement schemes for the conventional nonlocal signal, the (inverse) spin Hall effect signal, and the spin absorption signal are seen in Figs. 1(b)1(e) and the sample contacts are labeled in Fig. 1(a). Unless stated, measurements are performed at a temperature of $4.2 \mathrm{~K}$ via a cryostat. To measure the different spin transport signals, an alternating current of $1.0 \mathrm{~mA}$ with a frequency of $2221 \mathrm{~Hz}$ is applied (light blue). To generate this current, an alternating voltage of $5 \mathrm{~V}$ amplitude has been applied and a $5000 \Omega$ pre-resistor has been used before the sample to act as a current source. Since our nanowires have low resistances (some hundred $\Omega$ ), we can assume the same current for all temperatures and neglect the small variations of the sample resistance which are on the order of $200 \Omega$ for the central Py wire and less than $40 \Omega$ for the left and right Py wire and the Pt stripe. For the studied temperature dependence of the signals based on recipe 2, a pre-resistor of $1100 \Omega$ has been used in order to apply higher currents. However, since the probe configuration has been changed, the left and right Py wire as well as the Pt stripe act as injector electrodes which have resistances below $120 \Omega$ at room temperature and less than $80 \Omega$ at $4.2 \mathrm{~K}$. Therefore, the same current is assumed for all temperatures and small variations of the sample resistance with varying temperature are neglected.

To measure the conventional nonlocal signal for the two recipes we apply the current between the central Py wire and the left end of the $\mathrm{Cu}$ bridge as schematically shown in Fig. 1(b). The nonlocal voltage is then measured between the right $\mathrm{Py}$ wire and the right end of the $\mathrm{Cu}$ bridge. To measure the spin absorption signal [schematically shown in Fig. 1(e)], the current is applied between the central Py and the right end of the $\mathrm{Cu}$ bridge, and the nonlocal voltage is measured between the left Py wire and the left side of the $\mathrm{Cu}$ bridge. The signal is similar to the previous case, with the difference that now the spin current conduit passes over the Pt wire, whereas in the first case there is no wire in between the two Py electrodes.

To generate both the conventional nonlocal and the spin absorption signal, an external field is swept between $-100 \mathrm{mT}$ (a)
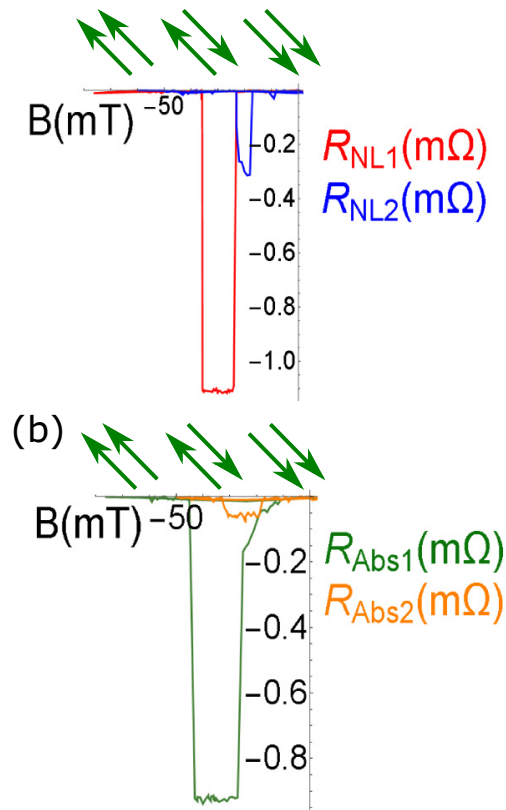

FIG. 2. Conventional nonlocal and spin absorption signal as a function of applied external field at $4.2 \mathrm{~K}$ for the different recipes and signals. To generate both signals, the external field is swept along the easy axes of the Py wires and the magnetization orientations of the probed Py wires are drawn as green arrows above the plots. (a) Conventional nonlocal signal of the sample fabricated by recipe 1 in red (current between contacts 6 and 1, signal between contacts 9 and 10) and recipe 2 in blue (current between contacts 7 and 1, signal between contacts 8 and 10). (b) Spin absorption signal of the sample based on recipe 1 in green (current between contacts 6 and 10, signal between contacts 3 and 1) and recipe 2 in orange (current between contacts 7 and 10, signal between contacts 2 and 1).

and $+100 \mathrm{mT}$ parallel to the easy axes of the Py wires, as indicated in the figure. The nonlocal resistance $R_{\mathrm{NL}}$ and the (inverse) spin Hall effect resistance $R_{(\mathrm{i}) \mathrm{SHE}}$ are defined as the measured voltages, divided by the applied current. The error bars for the different temperature-dependent curves are calculated as $\Delta_{\text {tot }}=\sqrt{\left(\Delta_{\mathrm{AP}}\right)^{2}+\left(\Delta_{\mathrm{P}}\right)^{2}}$, with $\Delta_{\mathrm{AP}, \mathrm{P}}$ as the standard error of the signals for high and low spin signal states.

First, we compare the conventional nonlocal spin signals with spin current injection and detection in Py electrodes for the samples based on the two different recipes shown in Fig. 2(a). We observe a signal of $1.10 \pm 0.01 \mathrm{~m} \Omega$ for the sample based on recipe 1 (red curve) and a signal of $0.34 \pm 0.01$ $\mathrm{m} \Omega$ for the sample fabricated by recipe 2 (blue curve). We find that samples fabricated by recipe 1 consistently yield approximately a factor 3 higher spin signals than samples based on recipe 2. Since the electric charge current interface resistances are very similar for samples based on the different recipes (in the range $8-10 \mathrm{~m} \Omega$ with variations on the order of $10 \%$ between recipes, which is within the observed spread for different samples for a single recipe), these differences in the signals cannot be explained by different charge transport interface resistances. Additionally we emphasize that for all measurements, the different possible injector/detectors permutations have been checked, with a maximum variation of 
the different signals for the different configurations within one device of $25 \%$. In this work we always show the highest measured spin signals, which accounts for the different indicated probe configurations for the different samples based on the different recipes.

The difference in signals implies a significant difference in the spin current polarizations in the two cases. The injected spin polarization can be estimated based on measurements of the nonlocal signal for devices with a range of separations between the injector and detector, as we have previously done for the Py/Cu system [34]. The typical low-temperature injected spin polarization for $\mathrm{Py} / \mathrm{Cu}$ that we find for samples fabricated with recipe 1 is on the order of $30 \%-35 \%$ $[34,35]$. In the present study we choose for our devices a single injector-detector separation and hence we did not repeat the detailed analysis but rely on previous results with similarly fabricated samples. Furthermore, information concerning the difference in the injected spin polarization can be estimated for the two cases based on the difference in signals for a set separation given the fact that the nonmagnetic $\mathrm{Cu}$ spin conduit is identical. The key parameters for determining the size of the nonlocal signal for a given electrode separation are the spin diffusion lengths of the materials, the dimensions of the samples, and the aforementioned injected spin polarization. Traditionally the effective area for spin injection cannot be measured and is included in the interfacial spin polarization. Via a 1-dimensional model of the system [36], the measured sample dimensions and resistivities [37], and assuming a spin diffusion length in Py of $5 \mathrm{~nm}$ and $\mathrm{Cu}$ of $1 \mu \mathrm{m}$ at low temperature [14], the expected signal can be estimated. Assuming effective rectangular interfaces and an injected polarization of $35 \%$ the model predicts a signal of $1.1 \mathrm{~m} \Omega$ for recipe 1 , in excellent agreement with the experimentally determined value. Due to the slightly wider $\mathrm{Cu}$ bridge for recipe 2 the model predicts a reduction in the signal to $0.95 \mathrm{~m} \Omega$ which is, however, still much larger than the observed signal in this case. Therefore, to account for the reduction in signal for recipe 2 compared with recipe 1 we would have to assume a reduction of the injected spin polarization to $22 \%$. Note that a simple reduction in the area of the contact region between the $\mathrm{Py}$ and $\mathrm{Cu}$ as a result of defects from recipe 2 does not explain the results, since a reduction in the geometrical size of the injection region is known to in certain cases increase the signal, in contrast to what we observe [38]. Hence, this value of $22 \%$ can be considered a limiting value. This shows that the spin polarization of the injected current strongly depends on the recipe and that significant depolarization occurs in the case of recipe 2 due to spin memory loss.

Next we compare the spin absorption strength, which is a measurement of the conventional nonlocal spin signal after the (partial) spin current absorption by $\mathrm{Pt}$, for the samples based on the two recipes as shown in Fig. 2(b). Here we find even larger differences. For the sample based on recipe 1, the spin absorption signal is $0.90 \pm 0.01 \mathrm{~m} \Omega$ (green curve) and thus about $20 \%$ smaller than the conventional nonlocal signal. Hence, within our variations of $25 \%$ for the injector/detector configurations, we do not determine significant spin absorption at the $\mathrm{Pt} / \mathrm{Cu}$ interface for the sample fabricated by recipe 1 . For the sample based on recipe 2 , however, we observe a spin absorption signal of $0.08 \pm 0.01 \mathrm{~m} \Omega$ (orange curve), resulting in a reduction of $76 \% \pm 3 \%$ [calculated as $\left.1-\left(R_{\mathrm{Abs}} / R_{\mathrm{NL}}\right)\right]$ of the nonlocal signal from the case without the intermediate Pt electrode and therefore a large expected spin absorption by the Pt electrode. The determined results for the sample based on recipe 2 with a reduction of the signal of $76 \% \pm 3 \%$ for Pt as an absorber material with large spin-orbit coupling agrees well with findings in the literature, where the absorption of various SHE materials including $\mathrm{Pt}$ [32,39], CuBi [31], and AuTa [33] has been studied using this approach in lateral spin valves. However, what is surprising in our study is the lack of differences in the conventional nonlocal and the spin absorption signal for the sample based on recipe 1 , with seemingly better interfaces, which calls for further investigation. In order to understand the differences we next compare the spin absorption signals to the (inverse) spin Hall effect signals in the heavy metals.

\section{COMPARISON OF THE MEASURED (INVERSE) SPIN HALL EFFECT SIGNALS}

To measure the inverse spin Hall effect signal, the same applied current has been used and the field is swept as before along the easy axes of the Py injector. Since the generated charge current in the Pt stripe due to the iSHE is

$$
J_{\text {iSHE }} \propto J_{S} \times \sigma,
$$

the polarity of the generated charge current $J_{\mathrm{iSHE}}$ in the Pt due to the iSHE changes sign on changing the orientation $\sigma$ of the spin current $J_{s}$. In order to switch $\sigma$, the injector magnetization is reversed by sweeping the external magnetic field. The measurement scheme of the inverse spin Hall effect signal and the nonlocal signal for spin Hall effect spin current generation (termed spin Hall effect signal) is shown schematically in Figs. 1(c) and 1(d), respectively.

As in our previously used geometry [40], we have patterned the Pt stripe and the Py wire perpendicularly to each other which provides the maximum changes in the spin signal via sweeps of the external field parallel to the easy axis of the FM wire. As a result, the two nonlocal resistance levels corresponding to two magnetic states are stable at remanence. Furthermore, the required fields to fully saturate the magnetization are much lower compared to previous publications where the heavy metal and the magnetic wires were often all oriented parallel to each other $[31,32]$. As presented in Fig. 3(a), we find for the sample fabricated by recipe 1 an inverse spin Hall effect signal of $0.40 \pm 0.01 \mathrm{~m} \Omega$ (brown curve) and for the sample based on recipe 2 a signal of $0.08 \pm 0.01$ $\mathrm{m} \Omega$ (purple curve). The size of the signal for the sample based on recipe 2 is consistent with the measured spin signal in our previous work [40], where also recipe 2 has been used for the $\mathrm{Cu}$ bridge processing. This difference of the iSHE signals as compared with the difference of the spin absorption signals, however, is counterintuitive:

One would expect that a large reduction of the spin signal by spin absorption into the Pt stripe should be connected with a large (inverse) spin Hall effect signal in the Pt, if both signals are based on the same spin current. From these results, we conclude that the size of the so-called spin absorption signal is not only related to the intrinsic properties of the Pt electrode. Rather the $\mathrm{Pt} / \mathrm{Cu}$ interface properties are of key importance 

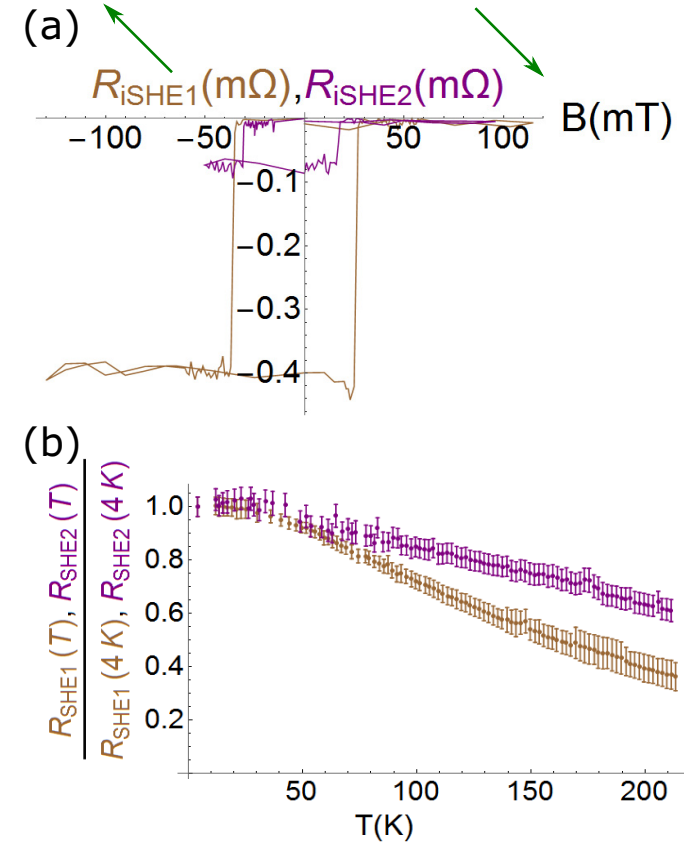

FIG. 3. (a) Inverse spin Hall effect signal as a function of the applied external field based on current injection between contact 6 or contact 7 and contact 10 in the case of recipe 1 and recipe 2, respectively. (b) Normalized spin Hall effect signal of the sample fabricated by recipe 1 (brown curve) and of the sample fabricated by recipe 2 (purple curve) in the temperature range between $4.2 \mathrm{~K}$ and $200 \mathrm{~K}$. Here the current is injected through the Pt through contacts 4-5 and detected as a nonlocal voltage between contact 6 or contact 7 and contact 10 in the case of recipe 1 and recipe 2, respectively. For temperatures between $50 \mathrm{~K}$ and $200 \mathrm{~K}$, a larger decrease of the normalized signal for the sample fabricated by recipe 1 is observed.

and additional contributions need to be taken into account which reduce the spin current without contributing to the inverse spin Hall effect, such as interface spin memory loss $[6,8,22,26]$.

To check this, we probe the normalized temperature dependence of the spin Hall effect for samples based on the two recipes, as shown in Fig. 3(b). Due to Onsager reciprocity $[41,42]$, varying the probe configuration does not change the signal, as confirmed in our measurements [40]. We find for temperatures between $50 \mathrm{~K}$ and $200 \mathrm{~K}$ a stronger decrease of the normalized spin Hall effect signal measured for the sample based on recipe 1 compared to recipe 2 . These differences can be explained by a strong temperature-independent reduction of the spin current, which partially masks the temperaturedependent contribution.

As a result, the decrease of the spin diffusion length in the $\mathrm{Cu}$ bridge with increasing temperature [14] as expected from Elliot-Yafet theory $[43,44]$, which is usually sufficient to explain the temperature behavior of the inverse spin Hall effect signal in this temperature range, is less dominant for samples fabricated by recipe 2 compared to samples fabricated by recipe 1 . If the $\mathrm{Pt} / \mathrm{Cu}$ interfaces for the two recipes are indeed significantly different (despite the very similar electric interface resistances), we expect that these differences should also clearly affect the temperature behavior of both

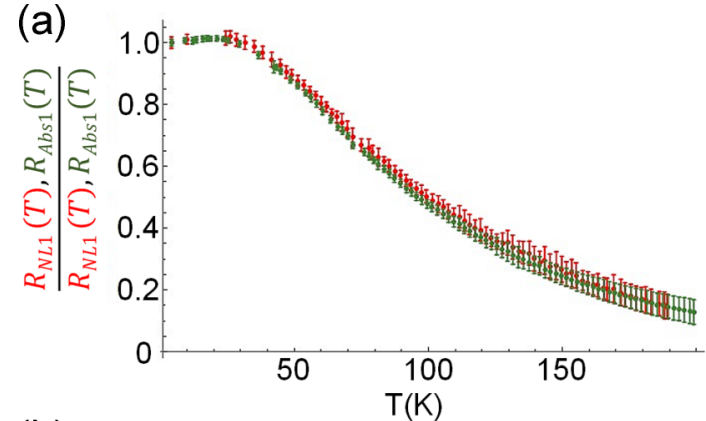

(b)

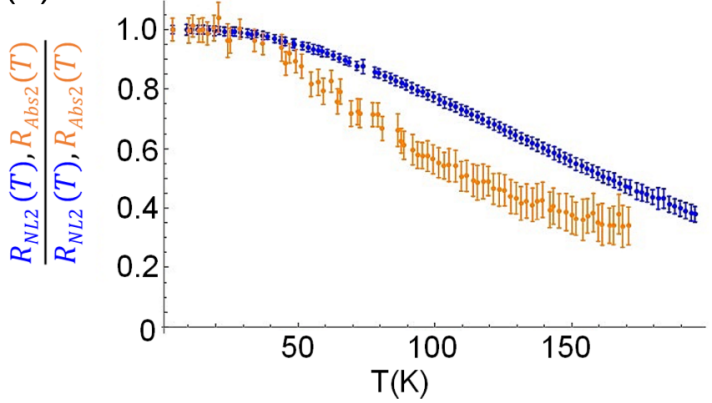

FIG. 4. Temperature dependence of the normalized conventional nonlocal signal and the spin absorption signal of the samples based on the different recipes. (a) Temperature dependence of the conventional nonlocal signal (NL, red curve) and the spin absorption signal (Abs, green curve) for the sample based on recipe 1. For the NL (Abs) measurement the current is applied between contacts 6-1 (6-10) and the signal is measured between contacts 9-10 (3-1). Within the error bars, the two curves are equivalent. (b) Temperature dependence of the conventional nonlocal signal (NL, blue curve) and the spin absorption signal (Abs, orange curve) for the sample fabricated by recipe 2 . For the NL (Abs) measurement the current is applied between contacts $8-10(2-1)$ and the signal is measured between contacts 3-1 (7-10). In the temperature range between $50 \mathrm{~K}$ and $200 \mathrm{~K}$ we find a stronger decrease of the spin absorption signal with increasing temperature.

the conventional nonlocal and the spin absorption signal for samples based on the different recipes. This is checked next.

\section{TEMPERATURE DEPENDENCE FOR THE CONVENTIONAL NONLOCAL AND THE SPIN ABSORPTION SIGNAL}

We now compare the temperature dependencies for the normalized conventional nonlocal and the spin absorption signal for both recipes, as plotted in Fig. 4. As expected from the comparison of the two signals at $4.2 \mathrm{~K}$, the signals of the sample based on recipe 1 are equivalent within the error bars. This is different for the sample based on recipe 2 , where a much stronger reduction of the spin absorption signal with increasing temperature is found. In previous publications, differences in the temperature behavior during the spin transport have been usually attributed to effects such as increased surface scattering [11,14] or the Kondo effect [45-47]. Since here the $\mathrm{Cu}$ conduit and the $\mathrm{Py} / \mathrm{Cu}$ interfaces are the same for a given recipe, all changes between the nonlocal signal and the spin absorption signal must be connected to the additional $\mathrm{Pt} / \mathrm{Cu}$ interface. 
These large differences in the temperature dependence for the two signals for the sample fabricated by recipe 2, combined with the identical temperature dependence of the signals of the sample based on recipe 1, support our previous findings concerning the differences in the (inverse) spin Hall effect signals. A significant amount of the generated spin current in the sample based on recipe 2 is lost due to spin-flip events at the interface and does not contribute to the (i)SHE signal.

\section{DIRECT IMAGING OF THE BURIED PT/CU AND PY/CU INTERFACES}

To probe the structural nature of the different relevant $\mathrm{Py} / \mathrm{Cu}$ and $\mathrm{Pt} / \mathrm{Cu}$ interfaces for devices made via the two recipes directly and thus to reveal the origin of the different results of the spin transport measurements, we additionally characterize the relevant interfaces via a special SEM technique. This technique allows for nondestructive buried interface imaging by employing a decelerated electron beam, as explained in detail in our previous work [17,18], where we also confirm the validity of the nondestructive imaging to assess the corresponding transport properties of devices in a local, spatially resolved manner, including MRAM junctions and $\mathrm{Fe} / \mathrm{GaAs}$-based lateral spin valves [19]. Note that in the devices based on tunneling or Schottky barrier interfaces the junction resistances are much higher than the full metallic systems we study here. While the imaging technique is able to detect the presence of defects at the buried interfaces equally for all cases, the presence of these defects may not always be directly evident from measurements of the junction resistance since in particular for the low-resistive interfaces the changes in the electrical signal can be small.

Individual SEM images have been taken using the commercial SEM "JEOL JSM 7800F". These images have been taken using different acceleration voltages, which are selected based on the results of a CASINO electron trajectory simulation [48]. The selective voltages for analyzing the $\mathrm{Cu} / \mathrm{Py}$ interface are $4.8 \mathrm{kV}$ and $5.1 \mathrm{kV}$, while for analyzing the $\mathrm{Cu} / \mathrm{Pt}$ interface, voltages of $4.9 \mathrm{kV}$ and $5.1 \mathrm{kV}$ have been used. An upper electron detector (UED) has been used in order to maximize the backscattered electron signal. The images, taken with different acceleration voltages, have been subsequently compared using a MATLAB script. In this manner, the contrast and alignment of the images has been readjusted and a processed image is generated.

We present here representative images of the $\mathrm{Pt} / \mathrm{Cu}$ and the central $\mathrm{Py} / \mathrm{Cu}$ interfaces, since the left and the right $\mathrm{Py} / \mathrm{Cu}$ interface show analogous results to the central one. As shown in Fig. 5, we observe significantly less inhomogeneities for samples fabricated by recipe 1 compared to samples based on recipe 2, which can be quantified via an "effective defect free interface area" (EA). The effective defect free interface area can be understood as the area without detectable inhomogeneities (marked by red boxes), divided by the total area.

The EA is selected by analyzing the contrast in the buried interface SEM images. First, the total area of the junction is defined based on the wire edges as revealed in the image. Within this area, regions with contrast varying from the wire background are assigned to defects and the ratio of the two areas defines the EA. The darker regions in the image mean
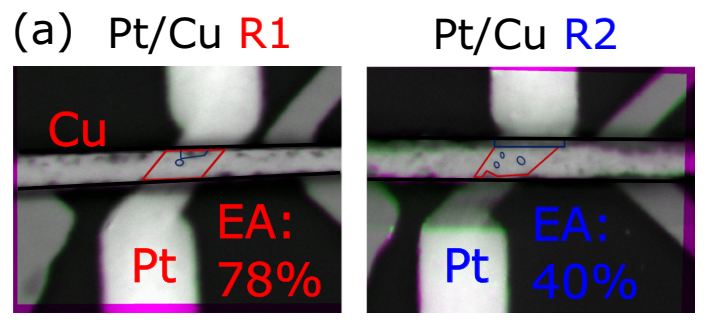

(b) C Py/Cu R1
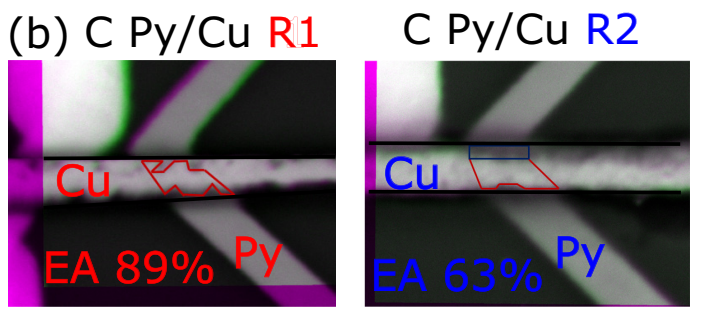

(c) EDX Cu R1

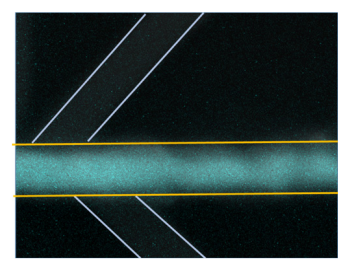

EDX Cu R2

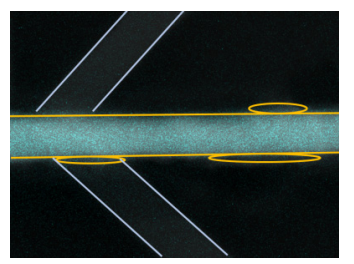

FIG. 5. (a), (b) Imaging of buried $\mathrm{Pt} / \mathrm{Cu}$ and $\mathrm{Py} / \mathrm{Cu}$ interfaces of samples based on the two different recipes. Regions marked by the red boxes indicate an effective area with a low amount of defects. Single defects within the red area are marked by blue circles. We find for samples based on recipe 2 shadow regions (marked by the blue boxes) at the top edge of the $\mathrm{Cu} / \mathrm{Pt}$ and the $\mathrm{Cu} / \mathrm{Py}$ interfaces, reducing significantly the effective defect-free interface area (EA). (c) Performed EDX measurements for the Cu content of the samples based on the different recipes. We find for samples based on recipe 2 additional $\mathrm{Cu}$ content at the edges of the $\mathrm{Cu}$ wire (marked by the yellow ellipses), consistent with the lower quality of the interfaces of samples based on recipe 2 compared to samples fabricated by recipe 1 .

that fewer backscattered electrons are generated, while the brighter regions indicate more backscattered electrons generated. For example, the former could be related to voids while the latter to the presence of residual resist. Further details about the nature of the defects can be determined via EDX mapping, as shown in Ref. [18]. In the present study no distinction between different defect types is made when defining the EA, since it is unknown in which way different defects affect the spin transport and we consider only regions with darker contrast as defects based on a threshold gray-scale value using image processing software. The darker defect regions shown at the interface do not generate many backscattered electrons, which indicates these regions are far less conductive as compared with the remaining interfaces. Such contrast reveals the presence of defects and inhomogeneities that can additionally impact the spin transport across the interfaces. Since the electrons are spin polarized the reduced conductivity directly leads to a reduction in the spin transport across the interfaces at these positions. Additionally the defects can lead to an increased spin memory loss for the electrons that do cross the interface, reducing the 
effective spin polarization, and this spin memory loss is not necessarily directly proportional to the reduced conductivity that is probed by the imaging. Hence, our estimate provides a qualitative evaluation of the effective area contributing to the spin transport. We emphasize that these effective areas should not be mistaken as simply reflecting the contact area of the interfaces, since the nonlocal signal scales reciprocally with the contact area [38], which is not the case for the EA described here. Rather the EA corresponds to interface regions where effective spin transport can be expected.

There are numerous types of defects that could be caused during the device fabrication stage. For example, residual resist may remain on the device at the liftoff stage, interfacial roughness can develop during evaporation, impurities may be present, etc. However, not all the defects will significantly modify the resistivity. The increase of electrical resistance is mainly due to a nonconductive medium that appears at the interface (e.g., residual resist and voids). Since interfacial roughness can also be identified using the nondestructive SEM method, the defects which are identified in the images do not necessarily contribute to the electric interface resistance which is often dominated by low-resistive regions. Nevertheless, the regions with low electrical conductivity are still able to absorb spins and therefore they can have a measurable impact on the spin signals. Even for current components flowing in the thin layers parallel to the interface, defects at the interface can lead to spin flips and thus reduce the spin polarization of the current. Furthermore it has been found that different contributions to the electron scattering that generates the electrical resistivity, including different types of morphological and impurity defects, have strongly varying differences in their associated spin-scattering probabilities, and hence they can influence the spin transport to different degrees compared to their effect on the electrical resistivity [49].

Within a clean area, we mark single defects as blue circles. We find for samples based on recipe 1 much more homogeneous $\mathrm{Pt} / \mathrm{Cu}$ and $\mathrm{Py} / \mathrm{Cu}$ interfaces, resulting in a $78 \% \mathrm{EA}$ for the $\mathrm{Pt} / \mathrm{Cu}$ and a $89 \% \mathrm{EA}$ for the central $\mathrm{Py} / \mathrm{Cu}$ interface. In particular for samples based on recipe 2 we observe at the top $\mathrm{Pt} / \mathrm{Cu}$ and the top $\mathrm{Py} / \mathrm{Cu}$ edge a shadow region (marked as a blue box in the plots), which is also found at the left and right $\mathrm{Py} / \mathrm{Cu}$ interfaces. These shadow regions significantly reduce the determined effective area down to $40 \%$ for the $\mathrm{Pt} / \mathrm{Cu}$ and $63 \%$ for the central $\mathrm{Py} / \mathrm{Cu}$ interface, reducing the overall interface quality for samples fabricated by recipe 2 . Our results lend themselves to the explanation that significantly more spin relaxation at the interfaces is generated for samples based on recipe 2. While the exact value determined for the EA depends slightly on the choice of threshold value in defining the defects, a significantly larger EA is reproducibly found in the case of recipe 1 . Since the EA does not take into account the nature of the interfacial defects, a direct quantitative comparison with the transport results should not be expected. However the revealed differences in the interfaces due to the two recipes is reflected in both a lower (i)SHE signal and a larger reduction in the nonlocal signal for recipe 2 , compared to samples based on recipe 1 with better interfaces.

The worse interface quality for samples based on recipe 2 compared to samples based on recipe 1 is supported by energy-dispersive $\mathrm{x}$-ray spectroscopy (EDX) measurements, as presented in Fig. 5(c). While for samples fabricated by recipe 1 the EDX results are as expected based on the sample design, we find for samples based on recipe 2 some additional $\mathrm{Cu}$ content at the $\mathrm{Pt} / \mathrm{Cu}$ and $\mathrm{Py} / \mathrm{Cu}$ edges, which is marked with yellow ellipses in the plot. From these measurements we conclude that although the liftoff based on a double-layer resist (recipe 2) is significantly easier compared to the liftoff based on a single-layer resist (recipe 1), recipe 2 leads to $\mathrm{Cu}$ content at undesired positions, which is consistent with the lower quality of the interfaces of samples based on recipe 2 compared to samples fabricated by recipe 1 .

Our work demonstrates that the device performance depends significantly on the used patterning process and deposition conditions since they lead to different interface qualities, which are not apparent from conventional characterization. This has direct consequences for the determination of key transport parameters such as $\theta_{\mathrm{SH}}$ and $\lambda_{\mathrm{HM}}$, describing the HM influence on the spin absorption and the (inverse) spin Hall effect signal. We determine for samples based on recipe 2 the spin diffusion length and the spin Hall angle of $\mathrm{Pt}$ to be $3 \mathrm{~nm}$ and $0.16 \%$, respectively, at $4.2 \mathrm{~K}$. For samples fabricated by recipe 1 (assuming $R_{\mathrm{Abs} 1} / R_{\mathrm{NL} 1}=0.8$ ), we evaluate $\lambda_{\mathrm{HM}}$ to be $19 \mathrm{~nm}$ while $\theta_{\mathrm{SH}}$ is determined to be $1.6 \%$ at $4.2 \mathrm{~K}$ by using the same method as Sagasta et al. [32]. Hence, depending on the interface quality, there is an order of magnitude difference in the determined effective parameters, similar to the spread seen in the reports from different groups in the literature [7,28,29].

To explain the strong reduction of the spin absorption signal together with the low (i)SHE signal measured in samples based on recipe 2 , different possible contributions are considered. One possibility is that residual resist is present at the interface and the contamination and associated disorder in those regions lead to enhanced spin-flip scattering, leading to spin memory loss. Furthermore the structural disorder could modify the bonding and related spin transparency of the interface.

The situation is different for samples based on recipe 1, where we find that the interfaces are of significantly higher quality. Additionally in these samples, no differences of the temperature dependence between conventional nonlocal and spin absorption signal have been seen. These results, which reveal strong differences depending on the interface homogeneity, suggest that spin transparency and spin memory loss are crucial and must be taken into account to explain the surprisingly high (i)SHE signal and the surprisingly low spin absorption. Our results for these samples suggest that there is little spin memory loss at the $\mathrm{Pt} / \mathrm{Cu}$ interface for samples based on recipe 1 and therefore no significant reduction of the spin absorption signal compared to the conventional nonlocal signal, in contrast to samples based on recipe 2. However, it is important to note that the absence of spin memory loss does not necessarily directly lead to a large (i)SHE signal, depending on the transparency of the interface. Due to the finite spin diffusion length in $\mathrm{Pt}$, only the spin accumulation that has passed across the $\mathrm{Pt} / \mathrm{Cu}$ interface can be scattered and consequently contribute to the (inverse) spin Hall effect.

Based on the strong variations of the determined effective spin transport parameters of the different systems, we conclude that the spin absorption method faces challenges 
for robust determination of the spin Hall properties due to its strong sensitivity to the different interfaces. Experimental methods which are based on thin films and do not require a multistep liftoff process, e.g., spin pumping [24-26], spin torque ferromagnetic resonance [7], or spin-orbit torque measurements [22], might be more suitable for an accurate determination of $\theta_{\mathrm{SH}}$ and $\lambda_{\mathrm{HM}}$. While also in these measurements contributions such as spin transparency and spin memory loss need to be considered, possible problems with residual resist and other fabrication-related inhomogeneities at the interfaces are less relevant.

We furthermore conclude that in our samples in addition to the intrinsic properties of $\mathrm{Pt}\left(\lambda_{\mathrm{HM}}\right.$ and $\left.\theta_{\mathrm{SH}}\right)$, which are relevant to explain the device behavior, also the influence of the relevant interfaces and especially contributions such as spin transparency and spin memory loss play a decisive role. In particular characterization methods that can directly reveal the root-cause contributions to the loss of spin information at the interfaces are crucial for a proper determination of spin absorption and the (inverse) spin Hall effect. For a proper characterization of the interfaces, both temperature-dependent spin transport and buried interface imaging are necessary since the subtle differences that lead to different spin transport may not be revealed by electrical characterization alone. Especially the correlation between the fabrication method and the resulting interfaces characterized by buried interface imaging and spin transport measurements provides invaluable insights into the device performance and reveals the strong sensitivity of spin transport to the fabrication recipe. In order to maximize the spin signals, next to a careful tailoring of the relevant interfaces, material combinations with minimized spin memory loss and maximized spin transparency, e.g., by suitable band structure matching [50], are promising.

\section{INFLUENCE OF OTHER SAMPLE PARAMETERS}

The results for the $\mathrm{Py}-\mathrm{Cu}-\mathrm{Pt}$ spin valves, as presented above, reveal that subtle changes in the fabrication process modify the device interfaces in such a way as to significantly affect the spin transport in the systems. The question naturally arises as to whether these results are more widely valid for different materials systems and other aspects of the fabrication including the absorber material. It would be expected that in addition to the employed resist, other factors of the patterning, such as the employed electron beam acceleration voltage, should also affect the quality of the interfaces and thereby the spin transport. Finally, in order to test these points, we fabricate another set of devices as shown in Fig. 6(g). Here all electrodes, including the injector (Inj) and detectors (Det), but also the absorber (Abs) are fabricated from CoFe via UHV thermal evaporation, while the spin channel is fabricated from $\mathrm{Cu}$, as before. We fabricate two sets of samples based on the same recipes for the $\mathrm{Cu}$ bridge from before and additionally investigate the influence of different acceleration voltages for the electron beam (EHT) used in the patterning of the CoFe wires. We compare the nonlocal and absorption signal for the different cases as presented in Table I. As before, the exposure doses for the different cases have been optimized to achieve low ohmic $\mathrm{CoFe} / \mathrm{Cu}$ interfaces leading to variations in the widths of the different components on the order of (a) $30 \mathrm{kV}$ CoFe Recipe 1

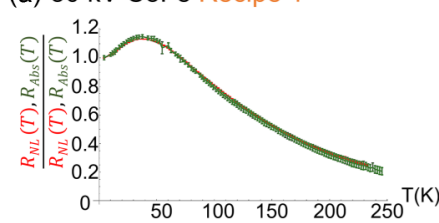

(c) $20 \mathrm{kV}$ CoFe Recipe 1

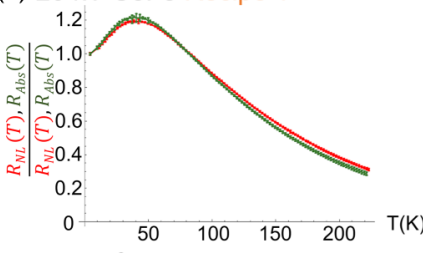

(e) $10 \mathrm{kV}$ CoFe Recipe 1

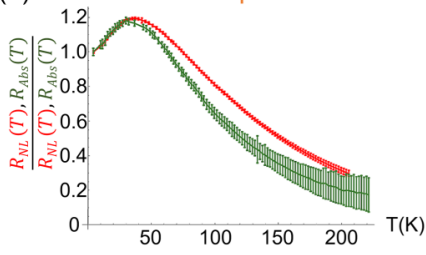

(b) $30 \mathrm{kV}$ CoFe Recipe 2

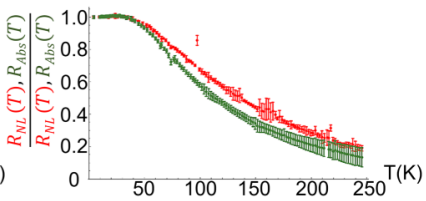

(d) $20 \mathrm{kV}$ CoFe Recipe 2

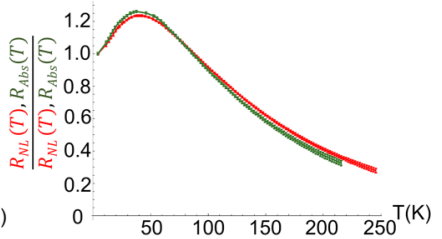

(f) $10 \mathrm{kV}$ CoFe Recipe 2

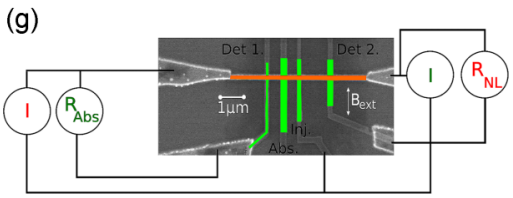

FIG. 6. (a)-(f) Temperature-dependent normalized conventional nonlocal (NL) and spin absorption signals (Abs) for $\mathrm{CoFe}-\mathrm{Cu}$ lateral spin valves with different $\mathrm{Cu}$ patterning recipes and different $\mathrm{CoFe}$ EHT patterning voltages. (g) Annotated scanning electron microscope image of the sample with the $\mathrm{Cu}$ conduit in orange and the $\mathrm{CoFe}$ electrodes in green. The probe configurations for the measurements of the nonlocal and absorption signal are indicated.

$10 \%$ [37]; however this does not account for the differences in signals for the different recipes and EHT cases.

In general the CoFe-based devices show higher signals compared to Py as a result of the higher effective spin current polarization [35]. It is evident that for a given EHT voltage both signals are higher in the case of recipe 1 for the patterning of the $\mathrm{Cu}$ bridge as compared to recipe 2 . This is consistent with the previous imaging results which directly revealed that recipe 2 leads to lower quality interfaces.

TABLE I. Influence of recipe and CoFe patterning EHT on the nonlocal and absorption signals for the two recipes for samples with all the electrodes $\mathrm{CoFe}$ and a $\mathrm{Cu}$ spin channel. The $30 \mathrm{kV}$ samples have $36 \mathrm{~nm}$ thick $\mathrm{CoFe}$ electrodes while the other samples have $25 \mathrm{~nm}$ CoFe electrodes.

\begin{tabular}{lcccc}
\hline \hline EHT CoFe & $\mathrm{Cu}$ Recipe & $\begin{array}{c}R_{\mathrm{NL}} \\
(\mathrm{m} \Omega)\end{array}$ & $\begin{array}{c}R_{\mathrm{Abs}} \\
(\mathrm{m} \Omega)\end{array}$ & $\begin{array}{c}1-\left(R_{\mathrm{NL}} / R_{\mathrm{Abs}}\right) \\
(\%)\end{array}$ \\
\hline $30 \mathrm{kV}$ & 1 & 4.8 & 2.4 & 50 \\
$30 \mathrm{kV}$ & 2 & 0.6 & 0.2 & 67 \\
$20 \mathrm{kV}$ & 1 & 3.4 & 2.3 & 32 \\
$20 \mathrm{kV}$ & 2 & 1.9 & 1.2 & 37 \\
$10 \mathrm{kV}$ & 1 & 1.2 & 0.4 & 68 \\
$10 \mathrm{kV}$ & 2 & 1.2 & 0.2 & 83 \\
\hline \hline
\end{tabular}


Furthermore we find that for a given recipe the EHT voltage for the CoFe patterning also strongly affects the measured signals. In the case of the conventional nonlocal signal, the highest signals are found for $30 \mathrm{kV}$ with the signal decreasing with decreasing voltage, whereas for the absorption signal the size of the signal depends both on the injected polarization and the amount of the spin current that is absorbed, resulting in a nonmonotonic dependence on the EHT. We therefore also find very different reductions of the signal between the two measurements, revealing the importance of all processing parameters for the interfaces and in turn the spin transport. Since the absorbing $\mathrm{CoFe}$ wire is relatively wide we see significant effective absorption in the case of both recipes, but this is larger for recipe 2, as before, where worse interfaces are expected, reinforcing the key role of interfacial spin relaxation in this case. Overall this means that, as before, the high nonlocal spin signals are not directly correlated with a large absorption of the spin current by the intermediate electrode. The temperature dependencies of the signals also reflect the differences in the interfaces, as presented in Fig. 6. On plotting together the two normalized signals for the different recipes and EHT voltages we find cases where the signals closely follow each other [panels (a), (c), and (d)] and other cases where significant differences in the temperature behavior are seen [panels (b), (e), and (f)]. When we compare this with the effective spin absorption for the different samples in Table I we find that when the effective absorption is $50 \%$ or less the temperature dependencies are similar whereas for larger effective absorption the temperature dependencies differ. Thus the differences in temperature can be here attributed to the contribution of the temperature dependence of the interfacial spin memory loss which is more significant at higher temperatures. Hence, depending on the fabrication mechanism this can be an important component of the signal, in addition to the transport in the conduit $[11,14,51]$ and the temperature dependence of the injection [40,52] and detection mechanisms [40]. Furthermore the existence of a maximum in the signal at low temperature is also seen to depend on the sample fabrication and thereby depending also on the interfaces of the device, supporting an interfacial component to this feature [45,47]. From these results we conclude that the size and temperature dependence of the spin transport signals are both strongly dependent on the defect structure of the interfaces for varying materials systems and that subtle changes to a wide range of fabrication parameters, that do not lead to significant changes of the device as assessed via conventional characterization techniques, nevertheless can lead to relevant changes to those interfaces that affect the spin transport.

\section{SUMMARY}

In summary, we have studied multiterminal $\mathrm{Pt}-\mathrm{Py}-\mathrm{Cu}-$ based lateral spin valves, which allow us to compare the spin absorption signal with the size of the (inverse) spin Hall effect for two fabrication recipes for the $\mathrm{Cu}$ conduit patterning. Very similar charge transport properties of the interfaces of the samples for the two recipes are found. However, we observe drastically different conventional nonlocal, spin absorption, and (inverse) spin Hall effect signals for samples based on the different recipes. For samples fabricated by the first recipe, where a single PMMA-resist layer has been used for processing, a very large (inverse) spin Hall effect signal is found but no significant spin absorption at the $\mathrm{Pt} / \mathrm{Cu}$ interface is observed. For samples based on the second recipe, where a dual MMA-PMMA resist layer has been used, we observe a low (inverse) spin Hall effect signal but find a reduction of the nonlocal signal of $76 \% \pm 3 \%$ at $4.2 \mathrm{~K}$. The impact of the recipes on the spin transport is confirmed for $\mathrm{CoFe} / \mathrm{Cu}$-based devices, revealing the origin of the differences is a general property of the different fabrication methods. Furthermore in these devices the EHT voltage used to pattern the CoFe wires is shown also to strongly influence the spin transport signals, demonstrating that the processing of the whole device is important.

These large differences of the signals for the different fabrication procedures are explained by interface spin loss and spin relaxation at the absorber $/ \mathrm{Cu}$ interface due to the different qualities of the interfaces. These effects crucially change the spin properties but do not significantly affect the electrical charge transport properties of our devices. By performing direct imaging of the buried $\mathrm{Pt} / \mathrm{Cu}$ and $\mathrm{Py} / \mathrm{Cu}$ interfaces, we observe significantly higher quality interfaces for samples based on recipe 1 compared to recipe 2 . Thus we are able to directly link the obtained spin signal with the imaged interface quality, which in turn is determined by the fabrication method including the different details of the patterning process. Our results clearly indicate that for a full understanding of spin transport through interfaces, not only the electric charge transport but additionally the interface spin transport properties are crucial and need to be tailored carefully. We find that good electrical transport across interfaces is not a good proxy for efficient spin transport.

One prominent consequence of this work is the fact that we determine strongly varying effective spin transport parameters of identically deposited $\mathrm{Pt}$ for the two fabrication recipes. For recipe 1 we find $\lambda_{\mathrm{HM}}=19 \mathrm{~nm}$ and $\theta_{\mathrm{SH}}=1.6 \%$ while for recipe 2 we find $\lambda_{\mathrm{HM}}=3 \mathrm{~nm}$ and $\theta_{\mathrm{SH}}=0.16 \%$. We conclude that the widely used spin absorption method is not always robust due to its strong sensitivity to the interface quality, which is not necessarily revealed in conventional electrical sample characterization or conventional imaging. Overall, the work demonstrates the vital role of interface structure in spin transport by correlating spin transport properties and the interface structural quality obtained from buried interface imaging, and we find that the interface quality is strongly dependent on the fabrication techniques.

\section{ACKNOWLEDGMENTS}

We acknowledge financial support of the SFB/TRR 173 Spin $+\mathrm{X}$ : spin in its collective environment (in particular project B02), funded by the Deutsche Forschungsgemeinschaft (DFG, German Research Foundation), Project No. 290396061/TRR173, as well as the Graduate School of Excellence Materials Science in Mainz (No. GSC266). This work was partially supported by JST CREST (No. JPMJCR17J5). 
[1] G. Binasch, P. Grünberg, F. Saurenbach, and W. Zinn, Enhanced magnetoresistance in layered magnetic structures with antiferromagnetic interlayer exchange, Phys. Rev. B 39, 4828 (1989).

[2] M. N. Baibich, J. M. Broto, A. Fert, F. Nguyen Van Dau, F. Petroff, P. Etienne, G. Creuzet, A. Friederich, and J. Chazelas, Giant Magnetoresistance of (001)Fe/(001)Cr Magnetic Superlattices, Phys. Rev. Lett. 61, 2472 (1988).

[3] E. B. Myers, D. C. Ralph, J. A. Katine, R. N. Louie, and R. A. Buhrman, Current-induced switching of domains in magnetic multilayer devices, Science 285, 867 (1999).

[4] D. C. Ralph and M. D. Stiles, Spin transfer torques, J. Magn. Magn. Mater. 320, 1190 (2008).

[5] A. Manchon, J. Železný, I. M. Miron, T. Jungwirth, J. Sinova, A. Thiaville, K. Garello, and P. Gambardella, Current-induced spin-orbit torques in ferromagnetic and antiferromagnetic systems, Rev. Mod. Phys. 91, 035004 (2019).

[6] J.-C. Rojas-Sánchez, N. Reyren, P. Laczkowski, W. Savero, J.-P. Attané, C. Deranlot, M. Jamet, J.-M. George, L. Vila, and H. Jaffrès, Spin Pumping and Inverse Spin Hall Effect in Platinum: The Essential Role of Spin-Memory Loss at Metallic Interfaces, Phys. Rev. Lett. 112, 106602 (2014).

[7] W. Zhang, W. Han, X. Jiang, S.-H. Yang, and S. S. P. Parkin, Role of transparency of platinum-ferromagnet interfaces in determining the intrinsic magnitude of the spin Hall effect, Nat. Phys. 11, 496 (2015).

[8] K. Dolui and B. K. Nikolić, Spin-memory loss due to spin-orbit coupling at ferromagnet/heavy-metal interfaces: Ab initio spindensity matrix approach, Phys. Rev. B 96, 220403(R) (2017).

[9] L. Zhu, D. C. Ralph, and R. A. Buhrman, Enhancement of spin transparency by interfacial alloying, Phys. Rev. B 99, 180404 (2019).

[10] C. Swindells, A. T. Hindmarch, A. J. Gallant, and D. Atkinson, Spin transport across the interface in ferromagnetic/nonmagnetic systems, Phys. Rev. B 99, 064406 (2019).

[11] E. Villamor, M. Isasa, L. E. Hueso, and F. Casanova, Contribution of defects to the spin relaxation in copper nanowires, Phys. Rev. B 87, 094417 (2013).

[12] W. Park, D. V. Baxter, S. Steenwyk, I. Moraru, W. P. Pratt, and J. Bass, Measurement of resistance and spin-memory loss (spin relaxation) at interfaces using sputtered current perpendicularto-plane exchange-biased spin valves, Phys. Rev. B 62, 1178 (2000).

[13] S. Yuasa, T. Katayama, M. Nvlt, Y. Suzuki, and T. Yori, Magnetoresistance in single $\mathrm{Fe}(001)$ ultrathin films, J. Appl. Phys. 83, 7031 (1998).

[14] T. Kimura, T. Sato, and Y. Otani, Temperature Evolution of Spin Relaxation in a NiFe/Cu Lateral Spin Valve, Phys. Rev. Lett. 100, 066602 (2008).

[15] T. Yang, T. Kimura, and Y. Otani, Giant spin-accumulation signal and pure spin-current-induced reversible magnetization switching, Nat. Phys. 4, 851 (2008).

[16] G. Mihajlović, J. E. Pearson, S. D. Bader, and A. Hoffmann, Surface Spin Flip Probability of Mesoscopic Ag Wires, Phys. Rev. Lett. 104, 237202 (2010).

[17] A. Hirohata, Y. Yamamoto, B. A. Murphy, and A. J. Vick, Non-destructive imaging of buried electronic interfaces using a decelerated scanning electron beam, Nat. Commun. 7, 12701 (2016).

[18] E. Jackson, M. Sun, T. Kubota, K. Takanashi, and A. Hirohata, Chemical and structural analysis on magnetic tunnel junctions using a decelerated scanning electron beam, Sci. Rep. 8, 7585 (2018).

[19] E. Jackson, Y. Wu, W. Frost, J.-Y. Kim, M. Samiepour, K. Elphick, M. Sun, T. Kubota, K. Takanashi, T. Ichinose, S. Mizukami, and A. Hirohata, Non-destructive imaging for quality assurance of magnetoresistive random-access memory junctions, J. Phys. D 53, 014004 (2019).

[20] J. E. Hirsch, Spin Hall Effect, Phys. Rev. Lett. 83, 1834 (1999).

[21] J. Sinova, S. O. Valenzuela, J. Wunderlich, C. H. Back, and T. Jungwirth, Spin Hall effects, Rev. Mod. Phys. 87, 1213 (2015).

[22] A. J. Berger, E. R. J. Edwards, H. T. Nembach, O. Karis, M. Weiler, and T. J. Silva, Determination of the spin Hall effect and the spin diffusion length of Pt from self-consistent fitting of damping enhancement and inverse spin-orbit torque measurements, Phys. Rev. B 98, 024402 (2018).

[23] A. Brataas, G. E. W. Bauer, and P. J. Kelly, Non-collinear magnetoelectronics, Phys. Rep. 427, 157 (2006).

[24] K. Ando, S. Takahasi, J. Teda, Y. Kajiwara, H. Nakayama, T. Yoshino, K. Harii, Y. Fujikawa, M. Matsuo, S. Maekawa, and E. Saitoh, Inverse spin-Hall effect induced by spin pumping in metallic system, J. Appl. Phys. 109, 103913 (2011).

[25] W. Zhang, V. Vlaminck, J. E. Pearson, R. Divan, S. D. Bader, and A. Hoffmann, Determination of the Pt spin diffusion length by spin-pumping and spin Hall effect, Appl. Phys. Lett. 103, 242414 (2013).

[26] X. Tao, Q. Liu, B. Miao, R. Yu, Z. Feng, L. Sun, B. You, J. $\mathrm{Du}, \mathrm{K}$. Chen, S. Zhang, L. Zhang, Z. Yuan, D. Wu, and H. Ding, Self-consistent determination of spin Hall angle and spin diffusion length in Pt and Pd: The role of the interface spin loss, Sci. Adv. 4, eaat1670 (2018).

[27] H. Nakayama, M. Althammer, Y.-T. Chen, K. Uchida, Y. Kajiwara, D. Kikuchi, T. Ohtani, S. Geprägs, M. Opel, S. Takahashi, R. Gross, G. E. W. Bauer, S. T. B. Goennenwein, and E. Saitoh, Spin Hall Magnetoresistance Induced by a Nonequilibrium Proximity Effect, Phys. Rev. Lett. 110, 206601 (2013).

[28] Y. Wang, P. Deorani, X. Qiu, J. H. Kwon, and H. Yang, Determination of intrinsic spin Hall angle in Pt, Appl. Phys. Lett. 105, 152412 (2014).

[29] M.-H. Nguyen, D. C. Ralph, and R. A. Buhrman, Spin Torque Study of the Spin Hall Conductivity and Spin Diffusion Length in Platinum Thin Films with Varying Resistivity, Phys. Rev. Lett. 116, 126601 (2016).

[30] Y. Niimi, Y. Kawanishi, D. H. Wei, C. Deranlot, H. X. Yang, M. Chshiev, T. Valet, A. Fert, and Y. Otani, Giant Spin Hall Effect Induced by Skew Scattering from Bismuth Impurities inside Thin Film CuBi Alloys, Phys. Rev. Lett. 109, 156602 (2012).

[31] Y. Niimi, H. Suzuki, Y. Kawanishi, Y. Omori, T. Valet, A. Fert, and Y. Otani, Extrinsic spin Hall effects measured with lateral spin valve structures, Phys. Rev. B 89, 054401 (2014).

[32] E. Sagasta, Y. Omori, M. Isasa, M. Gradhand, L. E. Hueso, Y. Niimi, Y. C. Otani, and F. Casanova, Tuning the spin Hall effect of Pt from the moderately dirty to the superclean regime, Phys. Rev. B 94, 060412(R) (2016).

[33] P. Laczkowski, Y. Fu, H. Yang, J.-C. Rojas-Sánchez, P. Noel, V. T. Pham, G. Zahnd, C. Deranlot, S. Collin, C. Bouard, P. Warin, V. Maurel, M. Chshiev, A. Marty, J.-P. Attané, A. Fert, H. Jaffrès, L. Vila, and J.-M. George, Large enhancement of the spin Hall effect in Au by side-jump scattering on Ta impurities, Phys. Rev. B 96, 140405(R) (2017). 
[34] N. Motzko, B. Burkhardt, N. Richter, R. Reeve, P. Laczkowski, W. Savero Torres, L. Vila, J.-P. Attané, and M. Kläui, Pure spin current-induced domain wall motion probed by localized spin signal detection, Phys. Rev. B 88, 214405 (2013).

[35] G. Zahnd, L. Vila, T. V. Pham, A. Marty, P. Laczkowski, W. S. Torres, C. Beigné, C. Vergnaud, M. Jamet, and J.-P. Attané, Comparison of the use of $\mathrm{NiFe}$ and $\mathrm{CoFe}$ as electrodes for metallic lateral spin valves, Nanotechnology 27, 035201 (2015).

[36] F. J. Jedema, M. S. Nijboer, A. T. Filip, and B. J. van Wees, Spin injection and spin accumulation in all-metal mesoscopic spin valves, Phys. Rev. B 67, 085319 (2003).

[37] A. Pfeiffer, Pure spin current transport and magnetic state manipulation in lateral spin valves, Ph.D. thesis, Johannes Gutenberg University Mainz, Mainz, Germany, 2020.

[38] T. Kimura, Y. Otani, and J. Hamrle, Enhancement of spin accumulation in a nonmagnetic layer by reducing junction size, Phys. Rev. B 73, 132405 (2006).

[39] V. T. Pham, L. Vila, G. Zahnd, A. Marty, W. Savero-Torres, M. Jamet, and J. P. Attané, Ferromagnetic/nonmagnetic nanostructures for the electrical measurement of the spin Hall effect, Nano Lett. 16, 6755 (2016).

[40] A. Pfeiffer, R. M. Reeve, and M. Kläui, Importance of spin current generation and detection by spin injection and the spin Hall effect for lateral spin valve performance, J. Phys.: Condens. Matter 30, 465802 (2018).

[41] T. Kimura, Y. Otani, T. Sato, S. Takahashi, and S. Maekawa, Room-Temperature Reversible Spin Hall Effect, Phys. Rev. Lett. 98, 156601 (2007).

[42] P. Jacquod, R. S. Whitney, J. Meair, and M. Büttiker, Onsager relations in coupled electric, thermoelectric, and spin transport: The tenfold way, Phys. Rev. B 86, 155118 (2012).

[43] R. J. Elliot, Theory of the effect of spin-orbit coupling on magnetic resonance in some semiconductors, Phys. Rev. 96, 266 (1954).
[44] Y. Yafet, G-factors and spin-lattice relaxation of conduction electrons, Solid State Phys. 14, 1 (1963).

[45] L. O'Brien, M. J. Erickson, D. Spivak, H. Ambaye, R. J. Goyette, V. Lauter, P. A. Crowel, and C. Leighton, Kondo physics in non-local metallic spin transport devices, Nat. Commun. 5, 3927 (2014).

[46] J. T. Batley, M. C. Rosamond, M. Ali, E. H. Linfield, G. Burnell, and B. J. Hickey, Spin relaxation through Kondo scattering in $\mathrm{Cu} / \mathrm{Py}$ lateral spin valves, Phys. Rev. B 92, 220420(R) (2015).

[47] K.-W. Kim, L. O’Brien, P. A. Crowell, C. Leighton, and M. D. Stiles, Theory of Kondo suppression of spin polarization in nonlocal spin valves, Phys. Rev. B 95, 104404 (2017).

[48] D. Drouin, A. R. Couture, D. Joly, X. Tastet, V. Aimez, and R. Gauvin, Casino V2.42-A: Fast and easy-to-use modeling tool for scanning electron microscopy and microanalysis users, Scanning 29, 92 (2007).

[49] J. D. Watts, L. O'Brien, J. S. Jeong, K. A. Mkhoyan, P. A. Crowell, and C. Leighton, Magnetic impurities as the origin of the variability in spin relaxation rates in $\mathrm{Cu}$-based spin transport devices, Phys. Rev. Materials 3, 124409 (2019).

[50] C. Lidig, J. Cramer, L. Weißhoff, T. R. Thomas, T. Kessler, M. Kläui, and M. Jourdan, Unidirectional Spin Hall Magnetoresistance as a Tool for Probing the Interfacial Spin Polarization of $\mathrm{Co}_{2} \mathrm{MnSi}$, Phys. Rev. Appl. 11, 044039 (2019).

[51] N. Motzko, N. Richter, B. Burkhardt, R. Reeve, P. Laczkowski, L. Vila, J.-P. Attané, and M. Kläui, Spin relaxation in $\mathrm{Cu}$ and Al spin conduits, Phys. Status Solidi A 211, 986 (2014).

[52] A. Pfeiffer, S. Hu, R. M. Reeve, A. Kronenberg, M. Jourdan, T. Kimura, and M. Kläui, Spin currents injected electrically and thermally from highly spin polarized $\mathrm{Co}_{2} \mathrm{MnSi}$, Appl. Phys. Lett. 107, 082401 (2015). 